\title{
Desarrollo y validación del cuestionario sobre condicionantes de éxito escolar en alumnos de secundaria
}

\section{Development and validation of a questionnaire about determinants of academic success in secondary school students}

\author{
Dr. Jordi LONGÁS MAYAYO. Profesor Asociado. Universidad Ramon Llull (jordilm@blanquerna.url.edul). \\ Dra. Elena CARRILLO ÁLVAREZ. Profesora Asociada. Universidad Ramon Llull (elenaca@blanquerna.url.edu). \\ Dr. Albert FORNIELES DEU. Serra Húnter fellow. Universitat Autonoma de Barcelona (albert.fornieles@gmail.com). \\ Dr. Jordi RIERA I ROMANI. Catedrático Educación. Universidad Ramon Llull (jordirr@rectorat.url.edu).
}

\section{Resumen:}

Introducción: El fracaso escolar supone un gran desafío tanto a nivel individual como a nivel social, por su impacto negativo en la cohesión social, la participación ciudadana, el desarrollo económico, la sostenibilidad e incluso su incidencia sobre los hábitos de salud. Diversos estudios han puesto de manifiesto la asociación entre el nivel socioeconómico familiar y los resultados escolares, hasta el punto de que dicho factor se ha considerado su principal determinante. No obstante, el hecho de atribuir el fracaso escolar esencialmente al contexto de pertenencia solo tiene valor descriptivo y podría estar dificultando el desarrollo de respuestas más adecuadas a este gran desafío. Una mejor comprensión sistémica e integral del fenómeno podría contribuir a un mejor abordaje de la problemática mediante el desarrollo de nuevas propuestas de política pública y de acción socioeducativa. Para ello, es necesario disponer de los instrumentos de medida adecuados que evalúen diferentes dimensiones de una manera integrada. Método: el objetivo del presente estudio es evaluar las propiedades psicométricas del Cuestionario sobre Condicionantes de Éxito Escolar (CCEE) en una muestra de 858 alumnos de secundaria residentes en contextos socio-demográficos vulnerables de 5 ciudades españolas. El cuestionario recoge ítems sobre aquellos factores que la literatura especializada ha vinculado de manera relevante con el rendimiento escolar, excluyendo aquellos que ya disponen de cuestionarios específicos validados. Resultados: el análisis de componentes principales dio lugar a 7 factores: contexto escolar, relaciones con los compañeros, expectativas personales, capital social, recursos TIC para el estudio, clima en clase y apoyo familiar. Los índices de ajuste muestran unas buenas propiedades del cuestionario. Conclusión: se concluye que el CCEE es un instrumento que posee propiedades psicométricas adecuadas para su uso

Fecha de recepción de la versión definitiva de este artículo: 19-07-2017.

Cómo citar este artículo: Longás Mayayo, J., Carrillo Álvarez, E., Fornieles Deu, A. y Riera i Romani, J. (2018). Desarrollo y validación del cuestionario sobre condicionantes de éxito escolar en alumnos de secundaria I Development and validation of a questionnaire about determinants of academic success in secondary school students. Revista Española de Pedagogía, 76 (269), 55-82. doi: https://doi.org/10.22550/REP76-1-2018-03 
en la identificación de factores que apoyan el éxito educativo en alumnos de secundaria.

Descriptores: éxito escolar, fracaso escolar, educación secundaria, pobreza, validación, cuestionario.

\section{Abstract:}

Introduction: School failure is a major challenge at individual and social levels, owing to its negative impact on social cohesion, citizen participation, economic development, and sustainability and also its impact on health habits. Various studies have shown the link between family socio-economic level and educational results, to the point that this factor has been regarded as their principal determinant. Nonetheless, the essentially attributing school failure to the students' milieu is only descriptive in value and could impede the development of more appropriate responses to this challenge. A better systemic and integral understanding of the phenomenon might contribute to a better approach to the problem through the development of new proposals for public policy and socio-educational actions. To this end, it is necessary to have appropriate measurement instruments that evaluate different dimensions in an integrated manner. Method: the objective of this study is to evaluate the psychometric properties of the Questionnaire on Determinants of Success at School (QDSS) in a sample of 858 secondary-school students resident in vulnerable socio-demographic settings from 5 Spanish cities. The questionnaire combines items about factors that specialist literature has significantly linked to educational attainment, excluding those that already have specific validated questionnaires. Results: the principal component analysis gave 7 factors: school environment, relationships with classmates, personal expectations, social capital, ITC study resources, climate in class, and family support. The goodness of fit indices show good properties for the questionnaire. Conclusion: the QDSS is an instrument that has appropriate psychometric properties for use in identifying factors that support educational success in secondary-school students.

Keywords: success at school, school failure, secondary education, poverty, validation, questionnaire.

\section{Introducción}

El estudio que presentamos se enmarca en una investigación que plantea una aproximación compleja y sistémica al fenómeno del éxito/fracaso escolar en España e investiga la importancia de los factores que apoyan la resiliencia educativa en alumnos que viven en contextos sociodemográficos vulnerables.

El término fracaso escolar, como opuesto al éxito escolar, es en sí mismo un concepto de contenido impreciso y ambiguo (Marchesi y Hernández, 2003). Habitualmente, se refiere a la proporción de individuos que no consiguen concluir los estudios obligatorios (Calero, Chois y Waisgrais, 2010; Escudero y Martínez, 2012) aunque a veces también se usa para designar a aquellos que fracasan en la secundaria postobligatoria, concepto que correspondería más claramente a la noción de abandono escolar temprano (Mena, Fernández-Enguita y Riviere , 2010). El término abandono escolar temprano hace refe- 
rencia al hecho de no alcanzar un nivel 3 de la Clasificación Internacional Normalizada de Educación (CINE), que en España se logra al finalizar la educación secundaria postobligatoria (Bachillerato o Formación Profesional). Según datos del Eurostat (2015), la tasa de abandono escolar temprano en España es del 21.9\% en los jóvenes entre 18 y 24 años, prácticamente el doble de la media comunitaria y una de las peores entre los países de la Unión Europea.

No acreditar las competencias mínimas valoradas y requeridas en nuestra sociedad supone enormes dificultades de inserción laboral y coloca a las personas de nuestro país en esta situación ante un mayor riesgo de exclusión social y vulnerabilidad por causa de la pobreza (Boada, Herrera, Mas, Miñarro, Olivella y Riudor, 2011; Gil-Flores, 2011; Jolonch, 2008; Sarasa y Sales, 2009). Datos recientes muestran que colectivos con bajos niveles de cualificación, sin estudios 0 educación primaria, tienen una tasa de paro alrededor del $48.9 \%$ y $35.2 \%$ respectivamente, cifra que disminuye a mayor nivel de estudios (INE, 2015a y 2015b).

Así, el fracaso escolar, en cualquiera de sus acepciones supone un gran desafío tanto a nivel individual como a nivel social, por su impacto negativo en la cohesión social, la participación ciudadana, el desarrollo económico, la sostenibilidad e incluso su incidencia en el desarrollo de hábitos de vida saludable y algunas problemáticas de salud pública (Belfield, 2008; Brunello y Da Paola, 2013; OMS, 2013). Tanto es así, que la Unión Europea, en su estrategia Europa 2020 fija el compromiso de reducir el abandono escolar prematuro a menos del 10\% en 2016 (Co- misión Europea, 2010, 2011). Sin embargo, alcanzar este hito es una ardua tarea y requiere una exhaustiva comprensión del fenómeno del fracaso escolar y las causas subyacentes (Brunello y Da Paola, 2013).

En este contexto, diversos estudios han puesto de manifiesto la asociación entre el nivel socioeconómico familiar y los resultados escolares (Bravo y Verdugo, 2007; Cordero, Crespo y Santín, 2010; Choi y Calero, 2013; Renée y Mcalister, 2011), hasta el punto de que dicho factor se ha considerado su principal determinante (Goldtorpe, 2000; Fernández-Enguita, Mena y Riviere, 2010; Ferrer y Albaigés, 2008). No obstante, dado que la correlación no implica causalidad y la no acreditación 0 el abandono prematuro de la escuela pueden venir motivados por la confluencia de circunstancias muy diversas, el hecho de atribuir el fracaso escolar esencialmente al contexto de pertenencia solo tiene valor descriptivo y, en nuestra opinión, podría estar dificultando el desarrollo de respuestas más adecuadas a este gran desafío (Smith, 2003). De esta manera, un mayor esfuerzo hacia una comprensión sistémica e integral del fenómeno podría contribuir a un mejor abordaje de la problemática mediante el desarrollo de nuevas propuestas de política pública y de acción socioeducativa (Civís y Longás, 2015; Longás, Cussó, de Querol y Riera, 2016).

Una de las principales dificultades para avanzar en esta dirección es la escasez de estudios suficientemente exhaustivos y metodológicamente adecuados para obtener datos, ya que, si bien existen investigaciones que de manera específica recogen evidencia empírica sobre la influencia en el fracaso y éxito escolar de factores 
individuales (Berliner, 2009), familiares (Hernando, Oliva y Pertegal, 2012; Jeynes, 2007), escolares (Flecha, 2002 y 2009) y comunitarios (Hatcher y Leblond, 2001), según nuestro conocimiento no se identifican investigaciones cuantitativas que integren todas las dimensiones.

Partiendo de una reciente revisión de la literatura realizada en el marco de este proyecto de investigación (Díaz-Gibson et al., 2017) y de los primeros resultados obtenidos a partir del análisis de historias de vida de alumnos en situación de vulnerabilidad y con trayectorias de éxito escolar (Longás et al., 2016), resumimos a continuación los principales condicionantes del fracaso y éxito escolar.

Atendiendo a la esfera individual resulta obvio que las capacidades intelectuales de cada alumno tienen relación con su éxito escolar. Pero un conjunto de condicionantes sociales, económicos y culturales del contexto familiar y escolar que inciden directamente sobre la educabilidad de la infancia y la adolescencia, limitan el peso de la inteligencia como factor explicativo del éxito (Bonal, Tarabini, Constans, Kliczkowski y Valiente, 2010). Ello permite entender que el grueso de la literatura sobre PISA (Calero et al., 2010; Cordero et al., 2010; Choi y Calero, 2013; Sánchez y García-Rodicio, 2006; entre otros) señale como variables más determinantes del éxito escolar:

a) El nivel socioeconómico familiar.

b) La procedencia de culturas minoritarias y familias inmigrantes.

c) La concentración elevada $(>20 \%)$ de alumnos de origen inmigrante en las escuelas.
Otros estudios más focalizados permiten ampliar el rango de factores condicionantes del éxito escolar de cada individuo, determinados también por las relaciones familiares. Destacan los siguientes factores y variables:

a) Psicoafectivos (autoestima, percepción de apoyo familiar, expectativas familiares positivas, motivaciones personales) (Carpenter et al., 2010; Longás et al., 2016; Núñez et al., 2013; Renée y Mcalister, 2011; Roman, Cuestas y Fenollar, 2008; Santana y Feliciano, 2011).

b) Personalidad, principalmente el desarrollo de la responsabilidad y capacidad de esfuerzo (Torres-Acosta, Rodríguez-Gómez y Acosta-Vargas, 2013) y la competencia social que se vincula a la socialización escolar y el desarrollo del propio capital social (Maccoby y Martin, 2006); biológicos, como un bajo peso al nacer o condiciones de salud carenciales (Berliner, 2009); o materiales, como el acceso a recursos culturales y tecnológicos suficientes (OCDE, 2013).

En contextos de pobreza se dan con más frecuencia carencias en estas variables (García Alegre, 2014) porque la situación de vulnerabilidad también condiciona el ambiente educativo de la familia, la implicación de los progenitores en la educación, su relación con la escuela y el acceso a recursos (Collet y Tort, 2011; Fernández-Enguita et al., 2010; Jeynes, 2007).

Entre las variables correspondientes al ámbito escolar destacan acciones educativas y organizativas orientadas a mejorar el éxito educativo, que incluyen estrategias de atención a la diversidad, intervención temprana preventiva, fomento de la colaboración entre docentes y promoción 
de la relación escuela-familia (Depalma, Matusov y Smith, 2009; Murillo y Roman, 2011; Pozo, Suárez y García-Cano, 2012; Rodríguez, Ríos y Racionero, 2012). En estudios recientes también emerge la importancia de la calidad docente, que incluye las competencias didácticas, orientadoras y la capacidad para generar vínculo y expectativas positivas (Hanushek y Woessmann, 2010; Rivkin et al., 2005). Alguna investigación da especial valor al apego 0 sentido de pertenencia de los alumnos a la escuela (Archambault, Janosz, Morizot y Pagani, 2009; Geddes, 2010; Marcus y Sanders-Reio, 2001). Esta adhesión favorecedora del éxito podría resultar de la integración en el grupo de iguales y el clima positivo - de convivencia y trabajo- en el aula y el centro que indaga el modelo PISA, junto a la percepción de apoyo por parte de los docentes (Roorda, Koomen, Spilt y Oort, 2011; Santana y Feliciano, 2011) y la construcción desde la experiencia escolar de expectativas de éxito en relación a la formación y el estudio (Pàmies, 2013). En relación a los recursos escolares, los estudios basados en PISA les otorgan poca significatividad (Calero y Escardíbul, 2007).

A su vez, y muy especialmente en contextos de bajo nivel socioeconómico, disponer de un entramado socioeducativo rico o capital social comunitario, algo imposible si no hay colaboración entre entidades educativas y sociales de la comunidad, favorece el éxito educativo (Bravo y Verdugo, 2007; Moliner, 2008; Pedró, 2012; Pozo, Suárez y García-Cano, 2012) por su impacto en la construcción de escenarios de oportunidad de aprendizaje y desarrollo social (Miller, 2016).

Tras nuestra revisión constatamos la necesidad de disponer de un instrumento que sistematice y unifique la recogida de datos sobre factores relevantes del éxito escolar para poder relacionarlos empíricamente y favorecer un análisis más holístico. Existen cuestionarios específicos validados y altamente reconocidos que pueden reportar información fiable sobre algunos condicionantes del éxito identificados en la literatura. En el marco de nuestra investigación consideramos la pertinencia de los siguientes:

a) Hollingshead Four-Factor Index of Socioeconomic Status (SES) para determinar el nivel socioeconómico (Canals, 2002; Hollingshead, 1975).

b) Escala Europea de Privación Material de los Hogares (Guio y Marlier, 2013) para identificar situaciones de vulnerabilidad.

c) NEO-FFI para valorar la personalidad responsable (Costa y McCrae, 2008).

d) VISA-TEEN (Costa-Tutusaus y Guerra-Balic, 2016) para valorar hábitos saludables y estado de salud. Para disponer de un instrumento específico que integre los otros factores relevantes en la literatura complementando los ya citados, el objetivo de este estudio es construir y validar un cuestionario dirigido a estudiantes de secundaria que permita evaluar de manera integrada condicionantes del éxito escolar a partir de sus percepciones.

\section{Método}

\subsection{Muestra}

La muestra del estudio se escogió mediante un muestreo de conveniencia y estuvo compuesta por 858 estudiantes de 
$4^{\circ}$ de ESO y $1^{0}$ de Bachillerato (2014-2015) de 29 centros educativos de las siguientes ciudades y sus áreas metropolitanas: Barcelona (32.2\%), Sevilla (13.8\%), Murcia (22.6\%), Tenerife (19.3\%) y Palma de Mallorca (12.1\%). De estos, un 50.2\% fueron hombres y un $49.4 \%$ mujeres, el $81.5 \%$ de los cuales cursaban $4^{\circ}$ de ESO en el curso 2014-15 y el $18.5 \%$ restante Bachillerato. El $67.8 \%$ de los participantes nació en 1999, el $20.7 \%$ en el año 1998, el $9.9 \%$ en el año 1997 y un $1.5 \%$ entre los años 19921996. La nota media del curso anterior, proporcionada por cada centro educativo, indicaba que solo el 4\% había obtenido una calificación de insuficiente; $27.4 \%$ suficiente; $32.5 \%$ bien; $24.8 \%$ notable y $11.3 \%$ excelente. Un $17 \%$ (5) centros de la muestra pertenecen a contextos socioeconómicos de nivel medio o medio alto, mientras que el resto son centros ubicados en contextos de bajo nivel socioeconómico considerando demográficos de renda media y paro. En la Tabla 1 se muestran las frecuencias y los porcentajes de las principales variables demográficas de la muestra.

TABLA 1. Características de la muestra.

\begin{tabular}{|lrr|}
\hline \multicolumn{1}{|c}{ Variables } & \multicolumn{1}{c|}{$\mathbf{N}^{\mathbf{o}}$} & \multicolumn{1}{c|}{$\%$} \\
TOTAL & $\mathbf{8 5 8}$ & $\mathbf{1 0 0}$ \\
\hline Ciudad & & \\
Barcelona & 276 & 32.2 \\
Sevilla & 194 & 22.6 \\
Murcia & 104 & 12.1 \\
Palma de Mallorca & 166 & 19.3 \\
Santa Cruz de Tenerife & 118 & 13.8 \\
\hline Año de nacimiento & & \\
1992-1996 & 13 & 1.5 \\
1997 & 84 & 9.9 \\
1998 & 175 & 20.7 \\
1999 & 574 & 67.8 \\
Sin datos & 12 & 1.4 \\
\hline Género & & \\
Hombre & 431 & 50.2 \\
Mujer & 424 & 49.4 \\
Sin datos & 3 & 0.3 \\
\hline Lugar de nacimiento & & \\
España & 715 & 83.3 \\
Fuera de España & 138 & 16.1 \\
Sin datos & 5 & 0.6 \\
\hline Años en la ciudad & & \\
>15 & 434 & 50.6 \\
11-15 & 267 & 31.1 \\
6-10 & 76 & 9.0 \\
0-5 & 70 & 8.3 \\
Sin datos & 11 & 1.3 \\
\hline
\end{tabular}

\begin{tabular}{|c|c|c|}
\hline Variables & $\begin{array}{l}N^{\circ} \\
858 \\
\end{array}$ & $\begin{array}{l}\% \\
100\end{array}$ \\
\hline \multicolumn{3}{|l|}{ Curso actual } \\
\hline $4^{\circ} \mathrm{ESO}$ & 699 & 81.5 \\
\hline 1-2 $2^{\circ}$ Bachillerato & 156 & 18.5 \\
\hline \multicolumn{3}{|l|}{ Nota media } \\
\hline Excelente & 34 & 11.3 \\
\hline Notable & 235 & 24.8 \\
\hline Bien & 279 & 32.5 \\
\hline Suficiente & 213 & 27.4 \\
\hline Insuficiente & 97 & 4.0 \\
\hline \multicolumn{3}{|l|}{ Nivel estudios padre } \\
\hline Sin Estudios & 66 & 7 \\
\hline Estudios primarios finalizados & 237 & 25.1 \\
\hline Bachillerato o CFGS inacabado & 119 & 12.6 \\
\hline Bachillerato o CFGS finalizado & 178 & 18.8 \\
\hline Estudios Univ. Incompletos & 56 & 5.9 \\
\hline Estudios universitarios $1^{\mathrm{er}}$ ciclo & 69 & 7.3 \\
\hline Estudios universitarios $2^{\circ}$ ciclo & 131 & 13.9 \\
\hline Sin datos & 89 & 9.4 \\
\hline \multicolumn{3}{|l|}{ Nivel estudios madre } \\
\hline Sin Estudios & 59 & 6.2 \\
\hline Estudios primarios finalizados & 240 & 25.4 \\
\hline Bachillerato o CFGS inacabado & 127 & 13.4 \\
\hline Bachillerato o CFGS finalizado & 181 & 19.2 \\
\hline Estudios Univ. Incompletos & 74 & 7.8 \\
\hline Estudios universitarios $1^{\mathrm{er}}$ ciclo & 83 & 8.8 \\
\hline Estudios universitarios $2^{\circ}$ ciclo & 121 & 12.8 \\
\hline Sin datos & 60 & 6.3 \\
\hline
\end{tabular}


Desarrollo y validación del cuestionario sobre condicionantes de éxito escolar ...

\begin{tabular}{|lrr|}
\hline \multicolumn{1}{|c}{ Variables } & \multicolumn{1}{c|}{$\mathbf{N}^{\mathbf{0}}$} & \multicolumn{1}{c|}{} \\
TOTAL & $\mathbf{8 5 8}$ & $\mathbf{1 0 0}$ \\
\hline Situación laboral padre & & \\
Activo & 700 & 74.1 \\
En paro & 115 & 12.2 \\
Pensionista & 52 & 5.4 \\
Sin datos & 78 & 8.3 \\
\hline
\end{tabular}

\begin{tabular}{|c|c|c|}
\hline Variables & $\begin{array}{l}\mathrm{N}^{\mathbf{o}} \\
858\end{array}$ & $\begin{array}{c}\% \\
100 \\
\end{array}$ \\
\hline \multicolumn{3}{|c|}{ Situación laboral madre } \\
\hline Activo & 624 & 66 \\
\hline En paro & 237 & 25.2 \\
\hline Pensionista & 24 & 2.7 \\
\hline Sin datos & 58 & 6.1 \\
\hline
\end{tabular}

Fuente: Elaboración propia.

\subsection{Instrumento}

El Cuestionario sobre Condicionantes de Éxito Escolar (CCEE) reúne ítems sobre aquellos factores que la literatura especializada ha vinculado de manera relevante con el rendimiento escolar, excluyendo aquellos que ya disponen de cuestionarios específcos validados. En consecuencia, además de las variables socio-demográficas, el cuestionario diseñado inicialmente pretendía evaluar los siguientes condicionantes del éxito escolar (Tabla 2): expectativas personales de éxito; inclusión (apego o sentido de per- tenencia de los alumnos) en el centro; relación de confianza con los compañeros; relación de confianza alumno-docentes; modelo de enseñanza según se apoya el proceso de aprendizaje; clima de clase/grupo de iguales; apoyo familiar; capital social; y acceso a recursos para el estudio. El formato de respuesta para todos los ítems es de escala tipo Likert de cinco puntos en función del grado de acuerdo con cada afirmación (A, muy de acuerdo/frase completamente cierta, valor 5; E, muy en desacuerdo/frase completamente falsa, valor 1).

TABLA 2. Relación de factores e ítems del cuestionario inicial.

\begin{tabular}{|l|l|}
\hline Factor (*) & Ítems \\
\hline $\begin{array}{l}\text { Expectativas } \\
\text { personales }\end{array}$ & $\begin{array}{l}\text { Q13 (Estudios universitarios), Q14 (Formación profesional), Q15 (No con- } \\
\text { tinuación estudios), Q16 (Futuro con estudios), Q17 (Dinero sin estudios), } \\
\text { Q18 (Motivación estudios), Q19 (Ascensor social), Q20 (Interés aprendi- } \\
\text { zaje), Q21 (Mejorar la sociedad), Q22 (Estudios por obligación), Q23 (No } \\
\text { alternativas estudio), Q24 (No razones estudio), Q25 (Pérdida de tiempo), } \\
\text { Q26 (Imposibilidad continuar estudios),Q27 (No defraudar familia). }\end{array}$ \\
\hline $\begin{array}{l}\text { Inclusión en el } \\
\text { centro educativo }\end{array}$ & $\begin{array}{l}\text { Q28 (Aprendizaje instituto), Q29 (Preparación útil), Q30 (Ganas ir cla- } \\
\text { se), Q31 (Esfuerzo en clase), Q32 (Interés trabajo clase), Q33 (Temas } \\
\text { poco interesantes), Q34 (Reto), Q35 (Sentido pertenencia instituto), Q36 } \\
\text { (Sentirse valorado instituto), Q37 (Buen trato instituto), Q38 (Partici- } \\
\text { pación instituto), Q39 (Exclusión instituto), Q40 (Satisfacción instituto). }\end{array}$ \\
\hline $\begin{array}{l}\text { Relación con } \\
\text { los compañeros }\end{array}$ & $\begin{array}{l}\text { Q41 (Facilidad hacer amigos), Q42 (Bullying), Q43 (Buena relación } \\
\text { compañeros), Q44 (Respeto compañeros), Q45 (Ayuda aprendizaje com- } \\
\text { pañeros), Q46 (Trabajo en equipo), Q47 (Resolución conflictos), Q48 (Re- } \\
\text { cibir ayuda compañeros). }\end{array}$ \\
\hline
\end{tabular}




\begin{tabular}{|l|l|}
\hline Factor (*) & Ítems \\
\hline $\begin{array}{l}\text { Confianza } \\
\text { alumno-docente }\end{array}$ & $\begin{array}{l}\text { Q49 (Valoración consejos profesores), Q50 (Preocupación profesores), } \\
\text { ticia profesores), Q54 (Escucha profesores), Q55 (Comodidad con pro- } \\
\text { fesores), Q56 (Castigos injustos profesores), Q57 (Cumplimiento pro- } \\
\text { mesas profesores), Q58 (Respeto profesores), Q59 (Sentirse valorado } \\
\text { profesores), Q60 (Hablar abiertamente profesores), Q61 (Altas expec- } \\
\text { tativas profesores). }\end{array}$ \\
\hline $\begin{array}{l}\text { Modelo de } \\
\text { enseñanza y } \\
\text { aprendizaje }\end{array}$ & $\begin{array}{l}\text { Q62 (No ayuda profesores), Q63 (Ayuda profesores), Q64 (Atención pro- } \\
\text { fesores), Q65 (No consejos profesores), Q66 (Explicación profesores), } \\
\text { Q67 (Altas expectativas grupo), Q68 (Capacidad reflexión). }\end{array}$ \\
\hline Clima en clase & $\begin{array}{l}\text { Q69 (No atención clase), Q70 (Ruido clase), Q71 (Ambiente clase), Q72 } \\
\text { (Inicio clase), Q73 (Asistencia clase), Q74 (Atención clase), Q75 (Debe- } \\
\text { res escolares), Q76 (Esfuerzo compañeros). }\end{array}$ \\
\hline Apoyo familiar & $\begin{array}{l}\text { Q77 (Comunicación familia tutor), Q78 (Implicación familia escuela), } \\
\text { Q79 (Apoyo estudios familia), Q80 (Ánimo familia estudios), Q81 (Ayu- } \\
\text { da deberes familia), Q82 (Seguimiento escolar familia), Q83 (Valoración } \\
\text { estudios familia), Q84 (Orgullo familia), Q85 (Comunicación familia } \\
\text { alumno). }\end{array}$ \\
\hline Capital social & $\begin{array}{l}\text { Q86 (Apoyo referente), Q87 (Participación barrio), Q88 (Integración ba- } \\
\text { rrio), Q89 (No referentes), Q90 (Valor actividades extraescolares), Q91 } \\
\text { (Red social escuela), Q92 (Red social barrio), Q137 (Actividades extraes- } \\
\text { colares), Q138 (Voluntariado). }\end{array}$ \\
\hline $\begin{array}{l}\text { Q132 (Ordenador hogar), Q133 (Ordenador personal), Q134 (Internet), } \\
\text { Q135 (Espacio estudio), Q136 (Libros). }\end{array}$
\end{tabular}

(*) No se han incorporado los ítems de datos sociodemográficos (Tabla 1).

Fuente: Elaboración propia.

El cuestionario final es el resultado de cuatro fases: (1) revisión bibliográfica, elaborada por el equipo investigador y triangulada con informaciones obtenidas en entrevistas con expertos para identificar las dimensiones a considerar, así como instrumentos ya validados que midieran estas dimensiones; (2) elaboración de la primera versión del cuestionario, (3) administración piloto a 47 estudiantes para validar la adecuación morfosintáctica de los ítems y su ajuste al constructo de partida, (4) validación psicométrica del instrumento, que es lo que reportamos en este artículo.

\subsection{Procedimiento de la validación psicométrica}

El estudio se llevó a cabo mediante la aplicación de un diseño de encuestas transversal. Este tipo de diseños permite la descripción de una población en un momento dado. Asimismo permite establecer relaciones entre variables y diferencias entre los distintos segmentos que configuran la población (León y Montero, 2015).

El cuestionario fue distribuido en los 29 centros participantes, a los que se desplazó un miembro del equipo de investi- 
gación, quién estuvo presente durante la respuesta del mismo. Previamente se había firmado un acuerdo de participación en el cual se estableció el compromiso de devolución de los resultados de cada centro educativo. En el estudio participaron todos los alumnos presentes en el momento de realizar el estudio y cuyos padres y/o tutores legales habían autorizado su participación mediante un documento de consentimiento informado pasivo.

\subsection{Análisis de datos}

Con el objetivo de verificar las propiedades psicométricas del cuestionario se realizaron los siguientes análisis:

a) Análisis de los ítems, a partir de los índices de dificultad y de la correlación ítem-total.

b) Con el objetivo de revisar la dimensionalidad del cuestionario realizamos un Análisis en Componentes Principales (ACP) con rotación Oblimin ya que los factores se mostraron parcialmente correlacionados (ver Tabla 5), por lo que no podemos asumir el supuesto de ortogonalidad que aconsejaría utilizar otras rotaciones habituales como, por ejemplo, Varimax (Thompson, 2004; Izquierdo, Olea y Abad, 2014). Con los 88 ítems originales, probamos diversas soluciones factoriales y seleccionamos aquella que mejor se ajustara a los criterios de adecuación factorial (peso factorial superior a .30 y porcentaje de varianza explicada), que proporcionara una alta consistencia interna, a la vez que parsimonia en la descripción de la estructura factorial, interpretabilidad y coherencia teórica de los resultados. c) Análisis de fiabilidad de los factores, a partir de los coeficientes de Alfa de Cronbach para los factores y la correlación entre factores latentes.

d) Análisis descriptivo de los resultados de la muestra, que incluye un estudio de los factores, considerando la media y la desviación típica, así como un estudio de las diferencias en función del género, años viviendo en la ciudad, nota media del curso anterior, país de nacimiento y nivel de estudios máximo conseguido por los progenitores. Para estos análisis se ha utilizado el test de t-student 0 ANOVA con contraste a posteriori usando la corrección de Tukey en función del tipo de variable.

Los análisis estadísticos se han realizado con el programa IBM SPSS 23.

\section{Resultados}

Tanto el índice de Kaiser-Meyer-Olkin de adecuación de la muestra $(\mathrm{KMO}=0.905)$ como el test de esfericidad de Barlett $\left(\chi^{2}=\right.$ 17891.378, $\mathrm{p}<0.001)$ mostraron la adecuación de la nueva estructura factorial tras realizar el ACP mediante rotación Oblimin utilizando las puntuaciones tipificadas (Z) de los ítems. La depuración de los ítems respondió a la aplicación de tres criterios de forma independiente: 1) eliminación de los ítems con saturaciones inferiores a 0.30 ; 2) eliminación de ítems cuya pertinencia a un determinado factor era conflictiva a nivel teórico; y 3) eliminación de ítems que disminuían la consistencia interna del factor (verificando la mejora del Alfa de Cronbach cuando el ítem era eliminado).

Así pues, suprimimos los ítems Q33 (temas poco interesantes), Q34 (reto), Q21 
(mejorar la sociedad) por mostrar saturaciones inferiores a 0.30; el ítem Q88 (integración barrio) que mostraba saturaciones similares en dos factores, y el ítem Q36 (sentirse valorado instituto) que saturaba en el factor Relación Compañeros y que por su contenido no era teóricamente justificable. Siguiendo criterios similares, también eliminamos los siguientes ítems: Q89 (no referentes), Q91 (red social), Q92 (red social sin estudios), Q14 (formación profesional), Q16 (futuro con estudios), Q17 (dinero sin estudios), Q27 (no defraudar a la familia), Q30 (ganas de ir a clase), Q32 (interés trabajo clase), Q35 (sentido pertenencia instituto), Q37 (buen trato instituto), Q38 (participación instituto), Q40 (satisfacción instituto), Q47 (resolución conflictos), Q52 (enfado profesores), Q135 (espacio tranquilo). Finalmente, los ítems Q20 (interés aprendizaje), Q29 (preparación útil), Q31 (esfuerzo en clase), Q74 (atención en clase) y Q75 (deberes escolares) tenían cargas similares en dos factores, por lo que los incorporamos en aquel con mayor coherencia conceptual.

Para la elección del número de factores a retener descartamos el criterio $\mathrm{K} 1$ de
Kaiser (autovalores superiores a uno) ya que sobreestima el número de factores a mantener (Izquierdo, Olea y Abad, 2014). Tras considerar diferentes criterios para la retención de factores, como el análisis paralelo (Horn, 1965; Buja y Eyuboglu, 1992), el gráfico de sedimentación (Cattell, 1966) y la significación y coherencia teórica de los factores, así como su interpretabilidad, la versión final quedó finalmente constituida por 65 ítems distribuidos en siete factores que explican el $42.91 \%$ de la varianza total, con valores que van desde el 17.94 al $2.91 \%$ de la varianza explicada por cada uno de ellos. En la Tabla 3 mostramos los pesos factoriales de cada ítem superiores a 0.30 y las comunalidades. En esta misma Tabla se puede apreciar como las comunalidades de todos los ítems superan el valor de .25 excepto en tres casos: Q138 ¿Participas como voluntario en alguna entidad o asociación? $\left(h^{2}=.116\right) ;$ Q133 ¿Dispones de ordenador personal 0 tablet personal (es decir, solo para ti)? $\left(h^{2}=.211\right)$ y Q19 Espero con los estudios conseguir un trabajo mejor que el de mis padres y familiares $\left(h^{2}=.226\right)$.

\section{TABLA 3. Análisis en componentes principales de las puntuaciones típicas de los 65} ítems, solución de 7 factores y rotación Oblimin.

\begin{tabular}{|l|c|c|c|c|c|c|c|c|}
\cline { 2 - 8 } \multicolumn{1}{c|}{} & \multicolumn{7}{c|}{ Componente } \\
\cline { 2 - 9 } \multicolumn{1}{c|}{} & I & II & III & IV & V & VI & VII & $\boldsymbol{h}^{2}$ \\
\hline $\begin{array}{l}\text { Q13 Como estudiante, mi aspiración } \\
\text { es acabar estudios universitarios. }\end{array}$ & & & -.507 & & & & & .296 \\
\hline $\begin{array}{l}\text { Q15 No preveo seguir estudiando } \\
\text { cuando acabe el instituto. }\end{array}$ & & & .461 & & & & & .252 \\
\hline $\begin{array}{l}\text { Q18 Me siento motivado y con } \\
\text { fuerzas para seguir estudiando en } \\
\text { el futuro. }\end{array}$ & & & -.620 & & & & & .554 \\
\hline
\end{tabular}




\begin{tabular}{|c|c|c|c|c|c|c|c|c|}
\hline & \multicolumn{8}{|c|}{ Componente } \\
\hline & I & II & III & IV & $\mathbf{V}$ & VI & VII & $\boldsymbol{h}^{2}$ \\
\hline $\begin{array}{l}\text { Q19 Espero con los estudios conse- } \\
\text { guir un trabajo mejor que el de mis } \\
\text { padres y familiares. }\end{array}$ & & & -.301 & & & & & .226 \\
\hline $\begin{array}{l}\text { Q20 El solo hecho de aprender } \\
\text { ya es un buen motivo para seguir } \\
\text { estudiando. }\end{array}$ & & & -.397 & & & & & .418 \\
\hline $\begin{array}{l}\text { Q22 Sigo estudiando porque estoy } \\
\text { obligado. }\end{array}$ & & & .615 & & & & & .421 \\
\hline $\begin{array}{l}\text { Q23 Sigo estudiando porque no sé } \\
\text { qué otra cosa hacer. }\end{array}$ & & & .488 & & & & & .333 \\
\hline $\begin{array}{l}\text { Q24 No encuentro razones impor- } \\
\text { tantes para seguir estudiando. }\end{array}$ & & & .689 & & & & & .494 \\
\hline $\begin{array}{l}\text { Q } 25 \text { En mi situación, continuar } \\
\text { estudiando me parece una pérdida } \\
\text { de tiempo. }\end{array}$ & & & .743 & & & & & .576 \\
\hline $\begin{array}{l}\text { Q26 En mi situación, continuar } \\
\text { estudiando me parece imposible. }\end{array}$ & & & .677 & & & & & .500 \\
\hline $\begin{array}{l}\text { Q28 En el instituto desarrollo } \\
\text { capacidades y hago aprendizajes } \\
\text { importantes. }\end{array}$ & & & -.340 & & & & & .405 \\
\hline $\begin{array}{l}\text { Q29 Las clases me aportan una } \\
\text { preparación útil para lo que me } \\
\text { propongo hacer en la vida. }\end{array}$ & & & -.323 & & & & & .420 \\
\hline $\begin{array}{l}\text { Q31 Me esfuerzo para dar lo mejor } \\
\text { de mí en clase. }\end{array}$ & & & -.326 & & & & & .406 \\
\hline $\begin{array}{l}\text { Q39 Me siento apartado en el } \\
\text { instituto. }\end{array}$ & & -.543 & & & & & & .442 \\
\hline $\begin{array}{l}\text { Q41 En el instituto hago amigos } \\
\text { fácilmente. }\end{array}$ & & .773 & & & & & & .596 \\
\hline $\begin{array}{l}\text { Q42 A menudo me siento insulta- } \\
\text { do, amenazado o acosado por los } \\
\text { compañeros del instituto. }\end{array}$ & & -.545 & & & & & & .442 \\
\hline $\begin{array}{l}\text { Q43 Me llevo bien con la mayoría } \\
\text { de compañeros en el instituto. }\end{array}$ & & .748 & & & & & & .611 \\
\hline $\begin{array}{l}\text { Q44 Me siento tratado con respeto } \\
\text { por la mayoría de compañeros del } \\
\text { instituto. }\end{array}$ & & .767 & & & & & & .652 \\
\hline $\begin{array}{l}\text { Q45 Con mis compañeros nos ayu- } \\
\text { damos mutuamente a aprender. }\end{array}$ & & .478 & & & & & & .437 \\
\hline
\end{tabular}




\begin{tabular}{|c|c|c|c|c|c|c|c|c|}
\hline & \multicolumn{8}{|c|}{ Componente } \\
\hline & I & II & III & IV & $\mathbf{V}$ & VI & VII & $\boldsymbol{h}^{2}$ \\
\hline $\begin{array}{l}\text { Q46 Tengo capacidad para trabajar } \\
\text { en equipo con mis compañeros. }\end{array}$ & & .500 & & & & & & .442 \\
\hline $\begin{array}{l}\text { Q48 En el instituto tengo compa- } \\
\text { ñeros que me ayudan cuando lo } \\
\text { necesito. }\end{array}$ & & .593 & & & & & & .460 \\
\hline $\begin{array}{l}\text { Q49 Cuando los profesores me dicen } \\
\text { que no haga alguna cosa, sé que tie- } \\
\text { nen una buena razón para decírmelo. }\end{array}$ & .558 & & & & & & & .391 \\
\hline $\begin{array}{l}\text { Q50 Los profesores se preocupan } \\
\text { por mí. }\end{array}$ & .776 & & & & & & & .615 \\
\hline $\begin{array}{l}\text { Q51 En el instituto hay profesores } \\
\text { con los que puedo hablar si tengo } \\
\text { problemas. }\end{array}$ & .582 & & & & & & & .421 \\
\hline $\begin{array}{l}\text { Q53 Los profesores siempre inten- } \\
\text { tan ser justos. }\end{array}$ & .720 & & & & & & & .539 \\
\hline $\begin{array}{l}\text { Q54 Los profesores siempre están } \\
\text { dispuestos a escuchar las ideas de } \\
\text { los alumnos. }\end{array}$ & .749 & & & & & & & .564 \\
\hline $\begin{array}{l}\text { Q55 Me siento seguro y cómodo con } \\
\text { mis profesores de este centro. }\end{array}$ & .796 & & & & & & & .668 \\
\hline $\begin{array}{l}\text { Q56 Los profesores castigan a los } \\
\text { alumnos sin ni siquiera saber lo } \\
\text { que ha sucedido. }\end{array}$ & -.478 & & & & & & & .323 \\
\hline $\begin{array}{l}\text { Q57 Mis profesores cumplen las } \\
\text { promesas que hacen. }\end{array}$ & .651 & & & & & & & .439 \\
\hline $\begin{array}{l}\text { Q58 Mis profesores me tratan con } \\
\text { respeto. }\end{array}$ & .693 & & & & & & & .558 \\
\hline $\begin{array}{l}\text { Q59 En clase me siento poco valo- } \\
\text { rado por mis profesores. }\end{array}$ & -.478 & & & & & & & .378 \\
\hline $\begin{array}{l}\text { Q60 En este centro se puede hablar } \\
\text { abiertamente con los profesores. }\end{array}$ & .717 & & & & & & & .542 \\
\hline $\begin{array}{l}\text { Q61 Mis profesores siempre espe- } \\
\text { ran que dé lo mejor de mí. }\end{array}$ & .511 & & & & & & & .412 \\
\hline $\begin{array}{l}\text { Q62 Si me quedo atrás con los con- } \\
\text { tenidos del curso no recibo ayuda de } \\
\text { mis profesores para recuperarlos. }\end{array}$ & -.471 & & & & & & & .307 \\
\hline $\begin{array}{l}\text { Q63 Mis profesores están dispuestos } \\
\text { a ofrecerme ayuda extra en relación } \\
\text { al trabajo de clase si lo necesito. }\end{array}$ & .660 & & & & & & & .417 \\
\hline
\end{tabular}




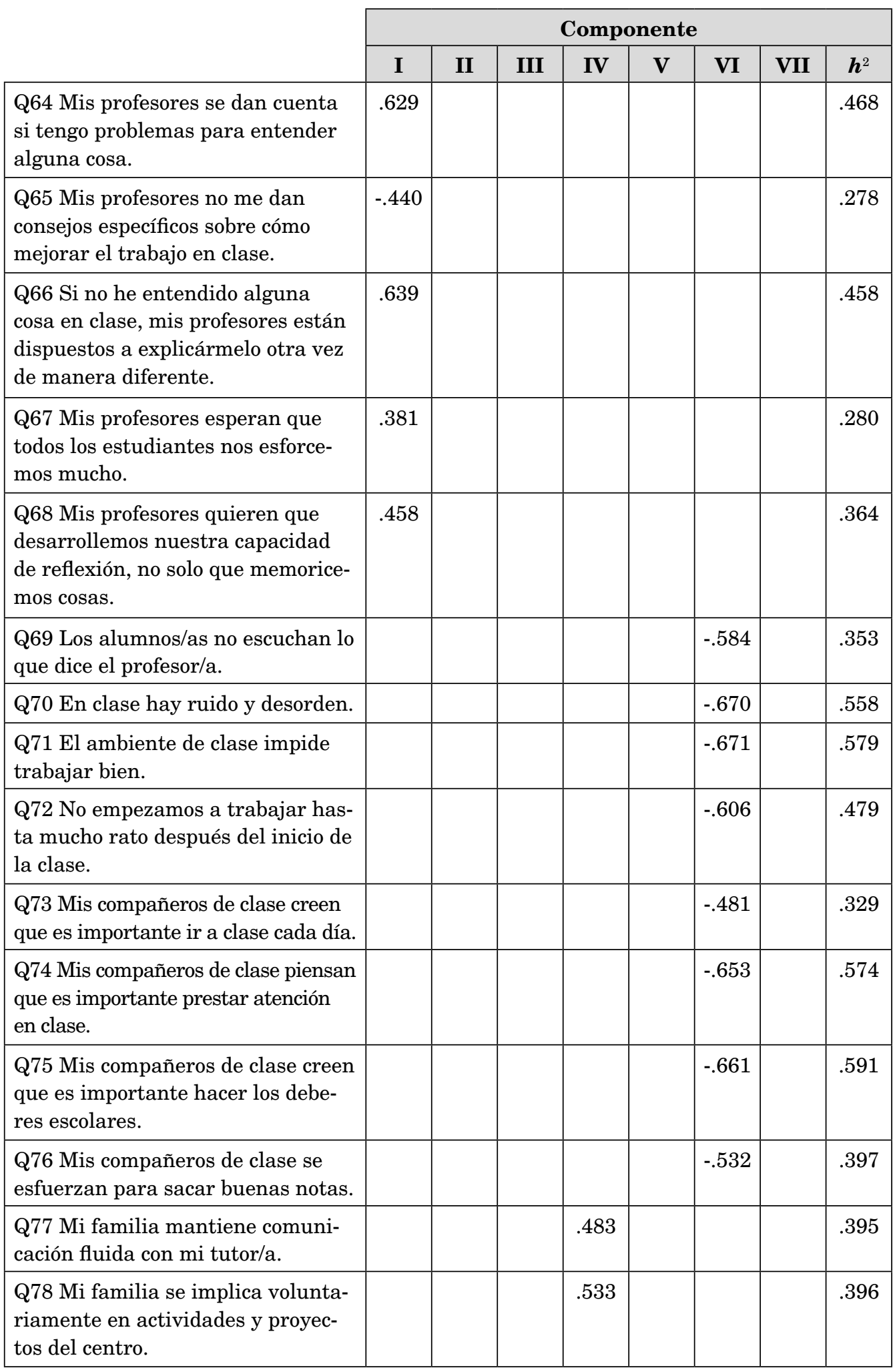




\begin{tabular}{|c|c|c|c|c|c|c|c|c|}
\hline & \multicolumn{8}{|c|}{ Componente } \\
\hline & $\mathbf{I}$ & II & III & IV & $\mathbf{V}$ & VI & VII & $\boldsymbol{h}^{2}$ \\
\hline $\begin{array}{l}\text { Q79 Como estudiante, no me siento } \\
\text { apoyado por mi familia. }\end{array}$ & & & & & & & -.571 & .396 \\
\hline $\begin{array}{l}\text { Q80 Mi familia me anima a esfor- } \\
\text { zarme en el instituto. }\end{array}$ & & & & & & & .716 & .570 \\
\hline $\begin{array}{l}\text { Q81 Cuando lo necesito, alguien } \\
\text { de mi familia me ayuda a hacer los } \\
\text { deberes escolares. }\end{array}$ & & & & & & & .657 & .487 \\
\hline $\begin{array}{l}\text { Q82 En mi familia nadie me pre- } \\
\text { gunta por mis exámenes o deberes. }\end{array}$ & & & & & & & -.551 & .345 \\
\hline $\begin{array}{l}\text { Q84 Siento que mi familia está } \\
\text { orgullosa de mí. }\end{array}$ & & & & & & & .629 & .547 \\
\hline $\begin{array}{l}\text { Q85 Hay personas de mi familia } \\
\text { con las que puedo hablar cuando lo } \\
\text { necesito. }\end{array}$ & & & & & & & .722 & .603 \\
\hline $\begin{array}{l}\text { Q86 Para mí ha sido muy impor- } \\
\text { tante el apoyo de un monitor/a, } \\
\text { educador/a, entrenador/a en los } \\
\text { últimos años. }\end{array}$ & & & & .364 & & & & .264 \\
\hline $\begin{array}{l}\text { Q87 Participo en actividades socia- } \\
\text { les y/o lúdicas en el barrio. }\end{array}$ & & & & .590 & & & & .383 \\
\hline $\begin{array}{l}\text { Q90 Actividades que realizo fuera } \\
\text { de la escuela me ayudan a mejorar } \\
\text { como persona. }\end{array}$ & & & & .469 & & & & .322 \\
\hline $\begin{array}{l}\text { Q132 ¿Cuántos ordenadores o } \\
\text { tablets hay en tu hogar? }\end{array}$ & & & & & .537 & & & .384 \\
\hline $\begin{array}{l}\text { Q133 ¿Dispones de ordenador } \\
\text { personal o tablet personal (es decir, } \\
\text { solo para ti)? }\end{array}$ & & & & & -.359 & & & .211 \\
\hline $\begin{array}{l}\text { Q134 ¿Dispones de internet en } \\
\text { casa? }\end{array}$ & & & & & -.422 & & & .246 \\
\hline $\begin{array}{l}\text { Q138 ¿Participas como voluntario } \\
\text { en alguna entidad o asociación? }\end{array}$ & & & & -.382 & & & & .161 \\
\hline $\begin{array}{l}\text { Q137 ¿Realizas actividades } \\
\text { extraescolares durante el curso } \\
\text { escolar? }\end{array}$ & & & & -.544 & & & & .329 \\
\hline$\%$ de varianza explicada & 17.94 & 5.40 & 5.175 & 4.53 & 3.55 & 3.40 & 2.92 & \\
\hline
\end{tabular}

Fuente: Elaboración propia.

Nota: $h^{2}=$ comunalidad. Se presentan las saturaciones $\geq .30$ 
El primer factor lo hemos denominado Contexto escolar, está compuesto por los ítems Q49, Q50, Q51, Q53, Q54, Q55, Q56, Q57, Q58, Q59, Q60, Q61, Q62, Q63, Q64, $\mathrm{Q} 65, \mathrm{Q} 66, \mathrm{Q} 67, \mathrm{Q} 68$ y valora las relaciones del alumno con el entorno escolar, explicando el $17.94 \%$ de la varianza. El segundo factor, denominado Relación con los compañeros, está compuesto por los ítems Q39, Q41, Q42, Q43, Q44, Q45, Q46, Q48, y valora las relaciones de confianza y ayuda entre compañeros, explicando el $5.40 \%$ de la varianza. El tercer factor se denomina Expectativas personales, está compuesto por los ítems Q13, Q15, Q18, Q19, Q20, Q22, Q23, Q24, Q25, Q26, Q28, Q29, Q31 y valora las expectativas asociadas al éxito en el estudio, explicando el 5.175\% de la varianza. El cuarto factor corresponde al Capital Social, está compuesto por los ítems Q77, Q78, Q86, Q87, Q90, Q137, Q138 y valora las relaciones y vínculos personales que pueden suponer un acceso a diferentes recursos, explicando el $4.53 \%$ de la varianza. El quinto factor, denominado Recursos TIC, está compuesto por los ítems Q132, Q133 y Q134 que valoran el acceso a tecnologías de la información y la comunicación que pueden favorecer el aprendizaje, explicando el 3.55\% de la varianza. El sexto factor se denomina $\mathrm{Cli}$ ma en clase, está compuesto por los ítems Q69, Q70, Q71, Q72, Q73, Q73, Q75, Q76 y valora la calidad de la convivencia en el grupo de iguales, explicando el $3.40 \%$ de la varianza. Por último, el séptimo factor lo hemos denominado Apoyo familiar, está compuesto por los ítems Q79, Q80, Q81, Q82, Q84, Q85 y valora la intensidad del apoyo de la familia directa al estudio y la escolarización, explicando el $2.92 \%$ de la varianza.

En la Tabla 4 se encuentran los principales resultados del análisis de los ítems. En concreto la descripción de los mismos (utilizando la media como medida de la dificultad), la correlación ítem-total del factor eliminado el ítem y el Alfa de Cronbach de cada factor en caso de eliminar el ítem. En la Tabla se puede observar que las correlaciones ítem-total son superiores a 0.3 en todos los casos salvo en los ítems 19, 137 y 138. Asimismo podemos apreciar que, excepto en el ítem 138, en ningún caso el Alfa aumenta eliminando el ítem.

Los resultados de análisis de fiabilidad (Tabla 5) muestran que la consistencia interna de los factores del cuestionario (puntuaciones tipificadas) es satisfactoria. Estos presentan unos valores de Alfa de Cronbach superiores a 0.8 para los factores $1,2,3$ y 6 , de 0.76 para el factor 7 e inferiores a 0.7 para los factores 4 y 5 , con una media de 0.77. En la misma Tabla también se muestran los descriptivos media y desviación estándar para los 7 factores, así como las correlaciones entre factores latentes. 
TABLA 4. Resultado del análisis de los ítems, a partir de los índices de dificultad y de la correlación ítem-total.
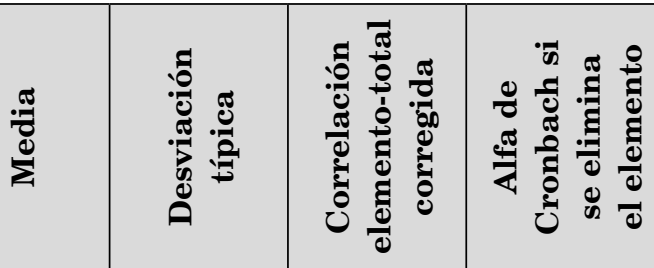

\section{FACTOR 1}

Q49 Valoración consejos profesores

2.147

Q50 Preocupación profesores

Q51 Hablar problemas profesores

2.199

1.071

Q53 Justicia profesores

Q54 Escucha profesores

Q55 Comodidad con profesores

Q56 Castigos injustos profesores

Q57 Cumplimiento promesas profesores

1.801

2.400

2.381

2.027

1.071

.

.539

(1)

.908

Q58 Respeto profesores

Q59 Sentirse valorado profesores

$\infty$

เ)

Q60 Hablar abiertamente profesores

Q61 Altas expectativas profesores

Q62 No ayuda profesores

Q63 Ayuda profesores

Q64 Atención profesores

Q65 Noconsejosprofesores_inv

Q66 Explicación profesores

Q67 Altas expectativas grupo

Q68 Capacidad reflexión

2.456

1.069

1.104

1.141

1.003

.72

\begin{tabular}{l|l}
.722 & .904 \\
\hline
\end{tabular}

\section{FACTOR 2}

\begin{tabular}{|l|c|c|c|c|}
\hline Q39 Exclusión instituto & 1.549 & .956 & .480 & .827 \\
\hline Q41 Facilidad hacer amigos & 1.925 & 1.010 & .629 & .806 \\
\hline Q42 Bullying & 1.401 & .792 & .427 & .831 \\
\hline Q43 Buena relación compañeros & 1.715 & .850 & .643 & .806 \\
\hline Q44 Respeto compañeros & 1.837 & .918 & .702 & .797 \\
\hline Q45 Ayuda aprendizaje compañeros & 2.176 & 1.013 & .525 & .822 \\
\hline Q46 Trabajo en equipo & 1.818 & .904 & .527 & .820 \\
\hline Q48 Recibir ayuda compañeros & 1.740 & .928 & .583 & .813 \\
\hline
\end{tabular}




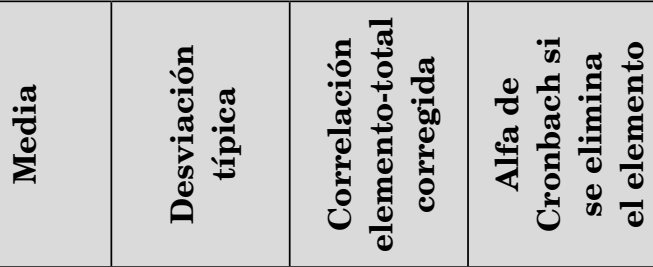

\section{FACTOR 3}

\begin{tabular}{|l|c|c|c|c|}
\hline Q13 Estudiosuniversitarios_inv & 3.911 & 1.434 & .335 & .804 \\
\hline Q15 Nocontinuaciónestudios & 4.787 & .716 & .318 & .797 \\
\hline Q18 Motivaciónestudios_inv & 4.086 & .963 & .602 & .774 \\
\hline Q19 Ascensorsocial_inv & 4.251 & .986 & .244 & .804 \\
\hline Q20 Interésaprendizaje_inv & 3.941 & 1.008 & .484 & .784 \\
\hline Q22 Estudiosporobligación & 3.923 & 1.152 & .487 & .783 \\
\hline Q23 Noalternativasestudio & 4.104 & 1.198 & .412 & .791 \\
\hline Q24 Norazonesestudio & 4.581 & .816 & .532 & .782 \\
\hline Q25 Pérdidadetiempo & 4.677 & .743 & .563 & .781 \\
\hline Q26 Imposibilidadcontinuarestudios & 4.610 & .816 & .464 & .787 \\
\hline Q28 Aprendizajeinstituto_inv & 3.881 & .944 & .481 & .784 \\
\hline Q29 Preparaciónútil_inv & 3.788 & 1.095 & .471 & .785 \\
\hline Q31 Esfuerzoenclase_inv & 3.539 & 1.028 & .415 & .790 \\
\hline
\end{tabular}

\section{FACTOR 4}

\begin{tabular}{|l|c|c|c|c|}
\hline Q77 Comunicación familia tutor & 2.995 & 1.283 & .392 & .582 \\
\hline Q78 Implicación familia escuela & 3.676 & 1.278 & .417 & .574 \\
\hline Q86 Apoyo referente & 2.683 & 1.381 & .318 & .607 \\
\hline Q87 Participación barrio & 3.672 & 1.398 & .456 & .558 \\
\hline Q90 Valor actividades extraescolares & 2.290 & 1.219 & .350 & .596 \\
\hline Q138 Voluntariado_inv_ajustado & 3.523 & 1.297 & .209 & .640 \\
\hline Q137_Tot_ajustada_inv & 3.870 & .933 & .284 & .616 \\
\hline
\end{tabular}

FACTOR 5

\begin{tabular}{|c|c|c|c|c|}
\hline Q132 Ordenador hogar & 3.262 & .889 & .516 & .264 \\
\hline Q133 Ordenadorpersonal_inv & 4.712 & .471 & .431 & .323 \\
\hline Q134 Internet_inv & 4.926 & .266 & .336 & .532 \\
\hline \multicolumn{5}{|l|}{ FACTOR 6} \\
\hline Q69 Noatenciónclase_inv & 2.949 & .903 & .399 & .801 \\
\hline Q70 Ruidoclase_inv & 2.789 & 1.101 & .560 & .779 \\
\hline Q71 Ambienteclase_inv & 3.151 & 1.145 & .534 & .783 \\
\hline
\end{tabular}




\begin{tabular}{|c|c|c|c|c|}
\hline & & 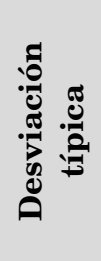 & 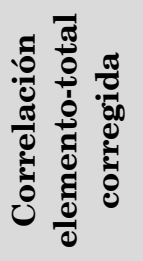 & 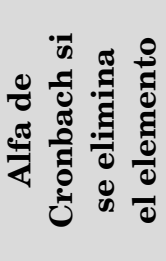 \\
\hline Q72 Inicioclase_inv & 3.162 & 1.190 & .483 & .791 \\
\hline Q73 Asistenciaclase_inv & 3.386 & 1.117 & .519 & .785 \\
\hline Q74 Atenciónclase_inv & 3.461 & 1.062 & .608 & .772 \\
\hline Q75 Deberesescolares_inv & 3.304 & 1.111 & .578 & .776 \\
\hline Q76 Esfuerzocompañeros_inv & 3.520 & 1.001 & .473 & .791 \\
\hline \multicolumn{5}{|l|}{ FACTOR 7} \\
\hline Q79 Apoyo estudios familia & 1.835 & 1.259 & .420 & .728 \\
\hline Q80 Ánimo familia estudios & 1.575 & .908 & .602 & .688 \\
\hline Q81 Ayuda deberes familia & 2.415 & 1.427 & .429 & .732 \\
\hline Q82 Seguimiento escolar familia & 1.847 & 1.205 & .385 & .737 \\
\hline Q84 Orgullo familia & 2.000 & 1.123 & .537 & .695 \\
\hline Q85 Comunicación familia alumno & 1.739 & 1.085 & .613 & .676 \\
\hline
\end{tabular}

Fuente: Elaboración propia.

$\infty$

เ)

$\infty$

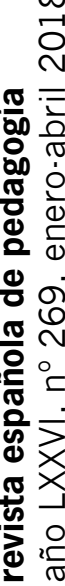

TABLA 5. Consistencia, descriptivos y correlaciones entre los factores del CCEE.

\begin{tabular}{|c|c|c|c|c|c|c|c|c|c|}
\hline & 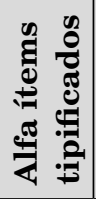 & $\Sigma$ & ํํำ & E & 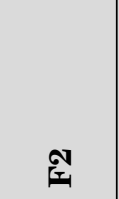 & 象 & 䏒 & 10 & $\underset{|c|}{0}$ \\
\hline F1: Contexto Escolar & .915 & 40.59 & 12.52 & & & & & & \\
\hline $\begin{array}{l}\text { F2: Relación } \\
\text { compañeros }\end{array}$ & .835 & 14.16 & 5.04 & $.426 * *$ & & & & & \\
\hline $\begin{array}{l}\text { F3: Expect. } \\
\text { personales }\end{array}$ & .814 & 54.08 & 7.14 & $-.448^{* * *}$ & $-.289 * *$ & & & & \\
\hline F4: Capital social & .634 & 22.70 & 4.94 & $.218^{* *}$ & $.216^{* *}$ & $-.111^{* *}$ & & & \\
\hline $\begin{array}{l}\text { F5: Recursos TIC } \\
\text { estudio }\end{array}$ & .687 & 12.90 & 1.29 & $-.015 .$. & $-.098^{*}$ & .022. & $-.118 * *$ & & \\
\hline F6: Clima en clase & .806 & 25.72 & 5.64 & $-.241 * *$ & $-.345^{* *}$ & $.147 * *$ & $-.078^{*}$. & $.022 .$. & \\
\hline F7: Apoyo familiar & .761 & 11.41 & 4.69 & $.386^{* *}$ & $.351^{* *}$ & $-.337 * *$ & $.227 * *$ & $-.143^{* *}$ & $-.131 * *$ \\
\hline
\end{tabular}

Fuente: Elaboración propia.

${ }^{*} p \leq 0.05 ; * * 00.01$ 
De este modo, el cuestionario adopta su forma final. Consta de 65 ítems y 7 factores: a) contexto escolar (19 ítems); b) relación con los compañeros (8 ítems); c) expectativas personales (13 ítems); d) capital social (7 ítems); e) recursos TIC para el estudio (3 ítems); f) clima en clase (8 ítems) y g) apoyo familiar (6 ítems). La puntuación de cada factor se obtiene por suma directa tras invertir la puntuación de los ítems: Q15, Q22, Q23, Q24, Q25, Q26, Q39, Q42, Q56, Q59, Q62, Q65, Q69, Q70, Q71 y Q72.

Los resultados de comparación entre grupos se pueden ver en la Tabla 6. En función del género existen diferencias significativas en los factores de contexto escolar, apoyo familiar y expectativas personales, siendo los hombres quienes muestran puntuaciones significativamente superiores en los dos primeros, mientras que la puntuación en cuanto a expectativas personales es mayor para las mujeres.

Respecto a la etapa educativa en el momento de responder (ESO, Bachillerato), las puntuaciones son significativamente mayores para los alumnos de bachillerato en capital social y clima en el aula, siendo superiores en recursos TIC para los alumnos de ESO.

Podemos observar diferencias en algunos factores también en función de la nota media del curso anterior. En alumnos cuya nota media es de insuficiente las puntuaciones son superiores significativamente en contexto escolar, mientras que para este mismo grupo son significativamente inferiores en expectativas personales. Por último, la puntuación en el factor 7, sobre apoyo familiar, fue significativamente más baja en aquellos alumnos cuya nota media fue de notable 0 excelente.

El hecho de haber nacido o no en España también genera diferencias significativas en algunos factores. El contexto escolar y recursos TIC muestra puntuaciones superiores para los nacidos en España, mientras que para los alumnos nacidos fuera de España el apoyo familiar obtiene puntuaciones significativamente más altas.

De la misma manera, los años de residencia en la ciudad también conllevan diferencias en cuanto a recursos TIC. En este caso, la puntuación de los que llevan 10-15 años es significativamente y superior de los que hace menos de 5 años que llegaron.

En cuanto al nivel máximo de estudios alcanzado por los progenitores, se dan diferencias significativas en los factores de recursos TIC y de apoyo familiar, aunque estas diferencias no son las mismas para el nivel educativo de la madre 0 del padre. En el primer caso, las puntuaciones de recursos TIC son significativamente mayores a partir de un nivel de estudios alcanzado de Bachillerato o CFGS finalizado por parte de la madre, mientras que en el caso paterno la puntuación solo es significativamente superior en el caso de haber acabado estudios universitarios de $2^{\circ}$ ciclo. En cuanto al apoyo familiar, las diferencias siguen un patrón menos estable, especialmente en el caso de la madre. Así, aquellos alumnos cuya madre alcanzó, como máximo, estudios universitarios de $1^{\circ} 02^{\circ}$ ciclo o bien cursó Bachillerato o CFGS (acabado o no), obtuvieron puntuaciones significativamente más ba- 
jas en apoyo familiar que el resto de grupos, mientras que en cuanto a educación paterna las diferencias en este factor solo son significativamente superiores en el caso de manifestar no tener estudios. La situación laboral de los padres no genera diferencias significativas en ninguno de los factores.

TABLA 6. Resultados de la comparación de medias en función de diferentes variables sociodemográficas.

\begin{tabular}{|c|c|c|c|c|c|c|c|}
\hline & \\
\hline & F1 & F2 & F3 & F4 & F5 & F6 & F7 \\
\hline \multicolumn{8}{|l|}{ Género } \\
\hline Hombre & $41.83^{* *}$ & 14.39 & $52.76^{* *}$ & 22.56 & 12.93 & 25.42 & $11.94^{* *}$ \\
\hline Mujer & $39.36^{* *}$ & 13.91 & $55.37 * *$ & 22.88 & 12.87 & 26.03 & $10.88 * *$ \\
\hline \multicolumn{8}{|l|}{ Curso } \\
\hline $4^{\circ} \mathrm{ESO}$ & 40.95 & 14.30 & 53.89 & $22.44^{* * *}$ & $12.95 * *$ & $25.20 * *$ & 11.48 \\
\hline $1^{\circ} \mathrm{BACH}$ & 38.99 & 13.55 & 54.88 & $23.91 * *$ & $12.67 * *$ & $28.03^{* *}$ & 11.11 \\
\hline \multicolumn{8}{|l|}{ País de nacimiento } \\
\hline España & $41.03^{*}$ & 14.10 & 53.97 & 22.73 & $12.97 * *$ & 25.67 & $11.15^{* *}$ \\
\hline Fuera España & $38.34^{*}$ & 14.33 & 54.77 & 22.49 & $12.58^{* * *}$ & 25.96 & $12.66^{* *}$ \\
\hline \multicolumn{8}{|l|}{ Años en la ciudad } \\
\hline$<5$ & 40.57 & 15.63 & 54.64 & 21.55 & $12.55^{* *}$ & 26.84 & 12.39 \\
\hline $6-9$ & 38.26 & 14.35 & 54.92 & 23.46 & 12.56 & 25.96 & 12.96 \\
\hline $10-15$ & 41.62 & 14.52 & 54.35 & 22.57 & $13.02 * *$ & 25.21 & 11.67 \\
\hline$>15$ & 41.00 & 14.95 & 52.65 & 21.57 & 12.76 & 23.33 & 12.05 \\
\hline \multicolumn{8}{|l|}{ Nota media curso } \\
\hline Insuficiente & 46.32 & 15.01 & 49.61 & 22.39 & 12.67 & 24.89 & 13.02 \\
\hline Suficiente & $41.45^{* *}$ & 14.53 & $53.14^{* *}$ & 22.60 & 12.79 & 25.52 & 11.82 \\
\hline Bien & $40.65^{* * *}$ & 14.34 & $53.94 * *$ & 23.11 & 12.80 & 25.89 & 11.79 \\
\hline Notable & $38.26 * *$ & 13.56 & $56.10 * *$ & 22.46 & $13.16^{* *}$ & 25.21 & $10.37^{* *}$ \\
\hline Excelente & $35.55^{* * *}$ & 12.14 & $59.09 * *$ & 22.55 & 12.90 & 24.51 & $8.44^{* *}$ \\
\hline \multicolumn{8}{|l|}{ Nivel estudios madre } \\
\hline Sin estudios & 40.42 & 15.09 & 51.85 & 13.18 & 12.32 & 25.85 & 13.72 \\
\hline Estudios primarios finalizados & 39.24 & 13.36 & 54.45 & 23.25 & 12.71 & 25.91 & 11.92 \\
\hline Bachillerato o CFGS inacabado & 40.38 & 14.35 & 54.83 & 22.39 & 12.80 & 25.78 & $11.29 * *$ \\
\hline Bachillerato o CFGS finalizado & 39.69 & 13.61 & 54.91 & 22.94 & $13.05^{* *}$ & 25.93 & $10.48 * *$ \\
\hline Estudios univ. incompletos & 41.24 & 14.22 & 55.13 & 22.29 & $13.06^{* *}$ & 25.66 & 1159 \\
\hline Estudios universitarios $1^{\mathrm{er}}$ ciclo & 41.45 & 13.27 & 54.18 & 22.29 & $13.05^{* *}$ & 26.18 & $10.55^{* *}$ \\
\hline Estudios universitarios $2^{\circ}$ ciclo & 43.73 & 14.04 & 53.13 & 21.71 & $13.30 * *$ & 25.19 & $10.53^{* *}$ \\
\hline
\end{tabular}


Desarrollo y validación del cuestionario sobre condicionantes de éxito escolar ...

\begin{tabular}{|c|c|c|c|c|c|c|c|}
\hline & F1 & F2 & F3 & F4 & F5 & F6 & F7 \\
\hline \multicolumn{8}{|l|}{ Nivel estudios padre } \\
\hline Sin estudios & 42.66 & 14.93 & 51.81 & 24.42 & 12.63 & 25.29 & $12.53^{* *}$ \\
\hline Estudios primarios finalizados & 39.41 & 13.69 & 53.74 & 22.62 & 12.68 & 26.30 & 11.63 \\
\hline Bachillerato o CFGS inacabado & 40.65 & 14.12 & 54.91 & 23.03 & 12.98 & 25.87 & 11.99 \\
\hline Bachillerato o CFGS finalizado & 39.75 & 13.81 & 54.89 & 22.04 & 13.05 & 25.69 & 10.54 \\
\hline Estudios univ. incompletos & 38.82 & 14.34 & 54.46 & 22.44 & 13.05 & 25.46 & 11.17 \\
\hline Estudios universitarios $1^{\mathrm{er}}$ ciclo & 41.96 & 13.53 & 54.05 & 22.03 & 13.12 & 25.83 & 10.55 \\
\hline Estudios universitarios $2^{\circ}$ ciclo & 42.96 & 14.21 & 54.21 & 22.27 & $13.20^{* * *}$ & 24.83 & 11.26 \\
\hline \multicolumn{8}{|l|}{ Situación laboral madre } \\
\hline Activo & 40.80 & 14.03 & 54.15 & 22.70 & 12.99 & 25.59 & 11.36 \\
\hline En paro & 40.47 & 14.37 & 53.76 & 22.88 & 12.69 & 25.90 & 11.52 \\
\hline Pensionista & 39.81 & 13.81 & 53.55 & 21.64 & 12.81 & 25.67 & 11.05 \\
\hline \multicolumn{8}{|l|}{ Situación laboral padre } \\
\hline Activo & 40.63 & 14.17 & 54.14 & 22.46 & 13.01 & 25.70 & 11.33 \\
\hline En paro & 39.58 & 13.95 & 53.87 & 23.08 & 12.43 & 25.97 & 11.18 \\
\hline Pensionista & 39.97 & 13.50 & 53.68 & 23.04 & 12.98 & 25.35 & 10.94 \\
\hline
\end{tabular}

Fuente: Elaboración propia.

NOTA: F1: Contexto Escolar; F2: Relación compañeros; F3: Expectativas personales; F4: Capital social; F5: Recursos TIC estudio; F6: Clima en clase; F7: Apoyo familiar.

${ }^{*} p \leq 0.05 ; * * p \leq 0.01$

\section{Discusión}

En el presente estudio, hemos analizado la estructura interna y las propiedades psicométricas del CCEE en una muestra de 858 estudiantes de $4^{\circ}$ ESO y $1^{\circ}$ Bachillerato (2014-15). El análisis de las variables socio-demográficas confirma la inexistencia de sesgos y señala que la muestra es representativa de diferentes áreas metropolitanas de España. Atendiendo al año de nacimiento, podemos indicar que el $89.5 \%$ de los participantes están dentro de promoción (no han repetido ningún curso) y solo el 4\% había obtenido una calificación media del curso de insuficiente. Los datos apuntan hacia un fenómeno habitual en secundaria, el abandono escolar prema- turo de alumnos cuando cumplen los 16 años y se encuentran fuera de promoción $\left(3^{\circ}\right.$ de ESO 0 1er trimestre de $4^{\circ}$ de ESO). Por ello consideramos que la muestra está más orientada al éxito y se ajusta bien al objetivo de nuestra investigación.

En relación a la validación del CCEE, tras realizar un ACP no ortogonal y barajar diferentes soluciones factoriales, la de 7 factores es la que mostró mayor coherencia teórica y mejor adecuación desde el punto de vista psicométrico. Siguiendo criterios estadísticos y racionales eliminamos 21 ítems de los 86 ítems diseñados inicialmente, quedando la versión final compuesta por 65 ítems. 
La versión que presentamos tiene unas buenas propiedades psicométricas proporcionando una estructura factorial teóricamente congruente y con una fiabilidad aceptable en los factores. Solo los factores 4 y 5 han obtenido una consistencia baja, sin embargo las hemos mantenido por su utilidad teórica, puesto que permiten considerar el capital social y los recursos TIC para su estudio como potenciales factores de éxito educativo (Bravo y Verdugo, 2007; Moliner, 2008; OCDE, 2013; Pedró, 2012; Pozo et al., 2012).

Nuestra estructura inicial de cuestionario contaba con 9 factores, resultado de la investigación teórica realizada. De ellos se han mantenido 6 factores después del análisis estadístico (Tabla 4): relación compañeros, expectativas personales, capital social, acceso a recursos para el estudio (centrado en acceso a recursos TIC), clima en clase y apoyo familiar. Y se ha generado 1 factor que hemos denominado contexto escolar que integra ítems de los iniciales factores teóricos: Confianza alumno-docente, Modelo de enseñanza-aprendizaje e Inclusión en el centro educativo. Aunque teóricamente podemos establecer las diferencias, puede suceder que los alumnos diferencien poco entre didácticas/metodologías docentes y su percepción de ayuda, acogida y buen trato por parte del docente. Por otra parte, el comportamiento de los ítems del factor inicial, inclusión en el centro educativo, claramente se ha polarizado entre la valoración general del centro y la valoración de la relación entre iguales. Resumiendo, sentir que el centro es útil o beneficioso y las buenas relaciones sociales con compañeros, explicarían el sentido de pertenencia al centro educativo.

Por consiguiente, consideramos que la estructura factorial del CCEE presenta un buen ajuste teórico, integrando en un solo instrumento los principales factores que se asocian como condicionantes o precursores del éxito escolar según la literatura (Longás et al., 2016). La composición final del instrumento permite reconocer la existencia de condicionantes escolares, familiares y comunitarios. La importancia de las expectativas personales como motor del éxito es, en sí misma, un factor de tipo individual que se construye tanto dentro como fuera de la escuela en la interacción con otras personas (Longás, et al., 2016). Del conjunto de factores cabe destacar el reconocimiento de la dimensión social o relacional del éxito escolar. Por eso, aunque el factor capital social no tenga una consistencia alta, la relación con los compañeros o el apoyo familiar, así como determinados ítems de contexto escolar, podrían también sumar a la reflexión sobre este interesante constructo como explicativo del éxito escolar (Coleman, 1988).

El comportamiento de los factores en función de las variables socio-demográficas también requiere una breve discusión. Diversos estudios que relacionan género y éxito escolar evidencian que las mujeres tienen menos abandono escolar y finalizan estudios superiores en mayor porcentaje (Calero et al., 2010). Nuestra investigación podría indicar alguna clave explicativa. Los factores condicionantes de éxito más altos en hombres con diferencia significativa son el contexto escolar y el apoyo familiar, ambos extrínsecos a la persona, 
mientras que en mujeres, el factor con valores significativamente más altos es el de expectativas personales. Este, de carácter intrínseco, podría condicionar el éxito en la trayectoria escolar de un modo más consistente (Torres-Acosta et al., 2013). Algo similar explicaría mejores resultados en algunos factores (capital social y clima en el aula) para los niveles educativos de bachillerato, en donde los alumnos tienen una proyección más definida y están integrados en niveles más exigentes que requieren mayor autonomía y responsabilidad (Roorda et al., 2011; Pàmies, 2013; Santana y Feliciano, 2011). Posiblemente las características de personalidad responsable, no medida en el CCEE, explicarían las diferencias en la percepción de apoyo familiar significativamente más bajo, algo que resulta imprescindible para tener éxito cuando se dan grados de poca autonomía personal o de inmadurez psicológica.

La valoración alta del contexto escolar por los alumnos con notas medias bajas puede ser un reconocimiento implícito de la ayuda escolar en su trayectoria, mientras que las expectativas personales bajas sería consecuencia de la trayectoria escolar de éxito reducido (posible causalidad inversa).

Las diferencias entre españoles y extranjeros muestran diferencias difíciles de explicar. Quizás las puntuaciones altas, a favor de los españoles, en contexto escolar y recursos TIC se deban a una mejor integración, mientras que el apoyo familiar alto en alumnos inmigrantes resulta un factor más determinante para quien debe integrarse en otra cultura y sistema educativo al del país de origen.
En relación al nivel de estudios de los progenitores los valores significativamente más altos en recursos TIC se corresponden con los niveles de formación más altos, que además de mayor sensibilidad pueden también indicar mayores recursos económicos. En cuanto al apoyo familiar es significativamente más alto con niveles de formación bajos en la madre 0 muy bajos en el caso del padre. Cabe valorar que en la percepción de los alumnos influya una mayor o menor presencia en el hogar de los progenitores, en relación con situaciones de paro o dificultades de conciliación laboral que tienen relación con los niveles de formación - algo que nuestros resultados parecen no apoyar, pero otros estudios sí (Collet y Tort, 2011; García Alegre, 2012), 0 indirectamente la influencia de los niveles de formación de los padres en los modelos de familia y el grado de desarrollo de la autonomía de los hijos.

\section{Conclusiones}

Las buenas propiedades psicométricas del CCEE permiten una evaluación amplia y parsimoniosa de cuáles son los principales factores condicionantes del éxito escolar en ambientes desfavorecidos.

Nuestros resultados deben ser considerados a la luz de ciertas limitaciones. Por una parte, únicamente disponemos de datos auto-informados, lo que puede suponer sesgos debidos a posible intencionalidad en las respuestas (deseabilidad social o magnificación de dificultades, por ejemplo), y por otra, la muestra estaba formada mayoritariamente por caucásicos lo cual puede mermar la generalización de los resultados a personas proce- 
dentes de otras culturas. También resulta limitante el modo de valorar el éxito escolar. Optamos por considerar la nota media del curso acabado como medida de la variable dependiente de este estudio, aunque otras informaciones como número de insuficientes por evaluación o notas medias de áreas instrumentales también se utilizan en otras investigaciones y resultan igualmente discutibles. Esta decisión nos permitió disponer de una información objetiva y fiable, facilitada por los centros educativos, ante la imposibilidad de disponer de pruebas de competencias estandarizadas de todos los componentes de la muestra.

Consideramos que la administración combinada del CCEE con otros instrumentos validados, referidos en este artículo, abre oportunidades para la investigación en torno al éxito escolar. Por ello ponemos a disposición de la comunidad científica un cuestionario que evalúa de forma rápida y fiable los factores que pueden condicionarlo.

\section{Referencias bibliográficas}

Archambault, I., Janosz, M., Morizot, J. y Pagani, L. (2009). Adolescent Behavioral, Affective and Cognitive Engagement in School: Relationship to Dropout. Journal of School Health, 79 (9), 408-415.

Belfield, C. (2008). The cost of early school-leaving and school failure. Washington, DC: World Bank.

Berliner, D. C. (2009). Poverty and Potential: Outof-School Factors and School Success. Boulder and Tempe: Education and the Public Interest Center \& Education Policy Research Unit. Recuperado de http://epicpolicy.org/publi- cation/poverty-and-potential (Consultado el 28/03/2015).

Boada, C., Herrera, D., Mas, E., Miñarro, E., Olivella, M. y Riudor, X. (Dir.) (2011). Informe sobre el risc de fracàs escolar a Catalunya. Collecció Estudis i Informes, 26. Barcelona: Consell de Treball, Econòmic i Social de Catalunya (Generalitat de Catalunya).

Bonal, X., Tarabini, A., Constans, M., Kliczkowski, F. y Valiente, O. (2010). Ser pobre en la escuela. Habitus de pobreza y condiciones de educabilidad. Barcelona: Miño y Dávila.

Bravo, M. y Verdugo, S. (2007). Gestión escolar y éxito académico en condiciones de pobreza. Revista Electrónica Iberoamericana sobre Calidad, Eficacia y Cambio en Educación, 5, 121-144.

Buja, A. y Eyuboglu, N. (1992). Remarks on Parallel Analysis. Multivariate Behavioral Research, 27 (4), 509-540.

Brunello, G. y Da Paola, M. (2013). The costs of early school leaving in Europe, IZA Discussion Paper, 7791. Bonn: IZA.

Calero, J. y Escardibul J. O. (2007). Evaluación de servicios educativos: el rendimiento en los centros públicos y privados medido en PISA2003. Hacienda Pública Española / Revista de Economía Pública, 183, 33-66.

Calero, J., Chois, K. y Waisgrais, S. (2010). Determinantes del riesgo de fracaso escolar en España: una aproximación a través de un análisis logístico multinivel aplicado a PISA-2006. Revista de Educación, número extraordinario 2010, 225-256.

Canals, J. (2002). Estudi de fiabilitat i validesa del CSI-4. Tesis Doctoral. Tarragona: Universitat Rovira i Virgili.

Carpenter, H., Cummings, C., Dyson, A., Jones, L., Laing, K., Oseman, D. y Todd, L. (2010). Extended services evaluation: end of year one report. Research report, dfe-rr016. London: Department for Education. Recuperado de 
https://www.gov.uk/government/uploads/system/uploads/attachment_data/file/182634/ DFE-RR016.pdf

Cattell, R. B. (1966). The Scree test for the number of factors. Multivariate Behavioral Research, 1 (2), 245-276.

Choi, A. y Calero, J. (2013). Determinantes del riesgo de fracaso escolar en España en PISA2009 y propuestas de reforma. Revista de Educación, 362, 562-593.

Civís, M. y Longás, J. (2015). La colaboración interinstitucional como respuesta al desafío de la inclusión socioeducativa. Análisis de 4 experiencias de trabajo en red a nivel local en Cataluña. Educación XXI, 18 (1), 213-236. doi:10.5944/educXX1.18.1.12318

Coleman, J. C. (1988). Social capital in the creation of human capital. American Journal of Sociology, 94, S95-S120.

Collet, J. y Tort, A. (2011). Famílies, escola i èxit. Millorar els vincles per millorar els resultats. Barcelona: Fundació Jaume Bofill.

Comisión Europea (2010). Europa 2020: Una estrategia para un crecimiento inteligente, sostenible e integrador. Bruselas: Comisión Europea. Recuperado de http://eur-lex.europa.eu/LexUriServ/LexUriServ.do?uri=COM:2010:2020:FIN:ES:PDF

Comisión Europea (2011). Comunicación de la comisión al parlamento europeo, al consejo, al comité económico y social europeo y al comité de las regiones. Abordar el abandono escolar prematuro: una contribución clave a la agenda europea 2020. Bruselas: Comisión Europea. Recuperado de http://ec.europa.eu/education/ school-education/doc/earlycom_es.pdf

Cordero, J. M., Crespo, E. y Santín, D. (2010). Factors affecting educational attainment: evidence from Spanish PISA 2006 results. Regional and Sectoral Economic Studies, 10, 55-76.

Costa, P. T. y McCrae, R. (2008). Inventario de personalidad NEO reducido de cinco facto- res (NEO-FFI) ( $3^{\mathrm{a}}$ Ed.). Madrid: TEA Ediciones.

Costa-Tutusaus, Ll. y Guerra-Balic, M. (2016). Development and psychometric validation of a scoring questionnaire to assess healthy lifestyles among adolescents in Catalonia. BMC Public Health, 16 (89).

Depalma, R., Matusov, E. y Smith, M. (2009). Smuggling Authentic Learning into the School Context: Transitioning From an Innovative Elementary to a Conventional High School. Teachers College Record, 111 (4), 934-972.

Escudero, J. y Martínez, B. (2012). Las políticas de lucha contra el fracaso escolar: iprogramas especiales 0 cambios profundos del sistema y la educación? Revista de Educación, número extraordinario 2012, 174-193.

Eurostat (2015). Europe 2020 education indicators in 2014. The EU is moving closer to its Europe 2020 goals on education. Recuperado de http://ec.europa.eu/eurostat/documents/2995521/6787423/3-20042015-BP-EN. pdf/b2f295ba-2e15-409c-bec9-91c4e49c5d32. (Consultado el 05/05/2015).

Fernández-Enguita, M., Mena, L. y Riviere, J. (2010). Fracaso y abandono escolar en España. Barcelona: Colección Estudios Sociales, Obra Social La Caixa.

Ferrer, F. y Albaigés, B. (2008). L'estat de l'esducació a Catalunya. Anuari 2006. Volum II. Barcelona: Fundació Jaume Bofill y Editorial Mediterrània.

Flecha, R., García, R., Gómez, A. y Latorre, A. (2009). Participación en escuelas de éxito: una investigación comunicativa del proyecto Includ-ed. Cultura y Educación, 21, 183-196.

Flecha, R. y L. Puigvert, L. (2002). La Comunidad de Aprendizaje: Una Apuesta por la igualdad educativa. REXE: Revista de Estudios Y Experiencias En Educación, 1, 11-20.

Geddes, H. (2010). El apego en el aula. Barcelona: Grao. 
Gil-Flores, J. (2011). Estatus socioeconómico de las familias y resultados educativos logrados por el alumnado. Cultura y Educación, 23 (1), 141-154.

Goldthorpe, J. (2000). On sociology. Oxford: Oxford University Press.

Guio, A. C. y Marlier, E. (2013). Alternative vs. current measures of material deprivation at EU level: What differences does it make? ImPRovE Discussion Paper, 13 (07). Antwerp: Improve.

Hanushek, E. y Woessmann, L. (2010). The Economics of International Differences in Educational Achievement. Working Paper 15949. Cambridge, MA: National Bureau of Economic Research.

Hatcher, R. y Leblond, I. (2001). Education Action Zones and Zones d'Education Prioritaires. Conferencia Travelling Policy / Local Spaces: Globalisation, Identities and Education Policy in Europe. Reino Unido: Keele University.

Hernando, A., Oliva, A. y Pertegal, M. A. (2012). Variables familiares y rendimiento académico en la adolescencia. Estudios de Psicología, 33, 51-56.

Hollingshead, A. A. (1975). Four-factor index of social status. Manuscrito inédito. New Haven, CT: Yale University.

Horn, J. L. (1965). A rationale and test for the number of factors in factor analysis. Psycometrika, 30, 179-185.

Instituto Nacional de Estadística. (2015a). Tasa de paro por nivel de formación alcanzado, sexo y grupo de edad. Encuesta de Población Activa. Resultados nacionales, 1er trimestre 2015. Madrid: INE. Recuperado de http://www.ine. es/jaxiT3/Tabla.htm?t=6393

Instituto Nacional de Estadística. (2015b). Tasa de riesgo de pobreza o exclusión social (estrategia Europa 2020) por nivel de formación alcanzado (personas de 16 y más años). Encuesta de Condiciones de Vida. Series Base 2013.
Resultados nacionales. Publicado el 26 Mayo de 2015. Madrid: INE. Recuperado de http:// www.ine.es/jaxiT3/Tabla.htm?t=4674\&L=0

Izquierdo, I., Olea, J. y Abad, F. (2014). Exploratory factor analysis in validation studies: Uses and recommendations. Psicothema, 26 (3), 395-400.

Jeynes, W. H. (2007). The relation between parental involvement and urban secondary school student academic achievement: A meta-analysis. Urban Education, 42, 82-110. doi. org/10.1177/0042085906293818

Jolonch, A. (2008). Exclusió social. Dels marges al cor de la societat. Barcelona: Fundació Lluís Carulla-ESADE, Col-lecció Observatori dels Valors.

León, O. G. y Montero, I. (2015). Métodos de investigación en Psicología y Educación (5 ${ }^{\mathrm{a}} \mathrm{Ed}$.). Madrid: McGraw Hill.

Longás, J., Cussó, I., de Querol, R. y Riera, J. (2016). Análisis de factores de apoyo a trayectorias de éxito escolar en la enseñanza secundaria en contextos de pobreza y vulnerabilidad social en España. Un estudio de casos múltiples. Revista de Estudios y Experiencias en Educación, 15 (28), 107-127.

Maccoby, E. y Martin, J. (2006). Socialization in the context of the family: Parent-child interaction. En Hetherington, E. (Ed.), Handbook of child psychology (pp. 1-101). New York: Wiley. Marchesi, A. y Hernández, C. (Coord.) (2003). El fracaso escolar. Una perspectiva internacional. Madrid: Alianza.

Marcus, R. F. y Sanders-Reio, J. (2001). The influence of attachment on school completion. School Psychology Quarterly, 16 (4), 427-444. Mena, L., Fernández-Enguita, M. y Riviere, J. (2010). Desenganchados de la educación: procesos, experiencias, motivaciones y estrategias del abandono y del fracaso escolar. Revista de Educación, número extraordinario 2010, 119-145. 
Miller, P. (2016). Liderazgo de intermediación en entornos complejos. Pedagogía Social Revista Interuniversitaria, 28, 17-24.

Moliner, O. (2008). Condiciones, procesos y circunstancias que permiten avanzar hacia la inclusión educativa: retomando las aportaciones de la experiencia canadiense. Revista Electrónica Iberoamericana Sobre Calidad, Eficacia y Cambio En Educación, 6, 27-44.

Murillo, J. F. y Román, M. (2011). School infrastructure and resources do matter: analysis of the incidence of school resources on the performance of Latin American students. School effectiveness and school improvement, 22 (1), 29-50.

OECD (2013).PISA2012Results:ExcellenceThrough Equity: Giving Every Student the Chance to Succeed (Volume II), PISA, OECD Publishing. doi: org/10.1787/9789264201132-en.

OMS (2013). Review of social determinants and the health divide in the WHO European Region: final report. Recuperado de http://www.euro. who.int/_data/assets/pdf_file/0004/251878/ Review-of-social-determinants-and-the-health-divide-in-the-WHO-European-Region-FINAL-REPORT.pdf?ua=1

Pàmies, J. (2013). The impact of groupings in school. Spaces of learning and sociability for Moroccan youth in Barcelona. Revista de Educación, 362, 133-158.

Pedró (2012). Políticas públicas sobre apoyo y refuerzo educativo: evidencias internacionales. Revista de Educación, número extraordinario 2012, 22-45.

Pozo, M. T., Suárez, M. y García-Cano, M. (2012). Logros educativos y diversidad en la escuela: hacia una definición desde el consenso. Revista de Educación, 358, 59-84.

Renée, M. y Mcalister, M. (2011). The strengths and challenges of community organizing as an education reform strategy: what the research says. Annenberg institute for school reform at brown university, Quincy, MA: Nellie Mae Education Foundation.

Rivkin, S., Hanushek, E. y Kain, J. (2005). Teachers, school and academic achievement. Econometrica, 73 (2), 417-458.

Rodríguez, H., Rios, O. y Racionero, S. (2012). Reframing Compensatory Education through Scientific Evidence. Inclusive Actions for Equality of Results. Revista de Educación, número extraordinario 2012, 67-87.

Roman, S., Cuestas, P. J. y Fenollar, P. (2008). An examination of the interrelationships between self-esteem, others' expectations, family support, learning approaches and academic achievement. Studies in higher education, 33 (2), 127-138.

Roorda, D., Koomen, H., Spilt, J. y Oort, F. (2011). The influence of affective teacher-student relationships on students' school engagement and achievement: A meta-analytic approach. Review of Educational Research, 81 (4), 493-529.

Sánchez, E. y García-Rodicio, H. (2006). Re-lectura del estudio PISA: qué y cómo se evalúa e interpreta el rendimiento de los alumnos en la lectura. Revista de Educación, número extraordinario 2006, 195-226.

Santana, L. E. y Feliciano, L. (2011). Parents' and teachers' perceived support, self-concept and decision making in high school students. Revista de Educación, 355, 493-519.

Sarasa, S. y Sales, A. (2009). Itineraris i Factors d'Exclusió Social. Barcelona: Ajuntament de Barcelona, Síndic de Greuges de Barcelona.

Smith, R. (2003). Research and Revelation: What Really Works. En P. Smeyers y M. Depaepe (Eds.), Beyond Empiricism: On Criteria for Educational Research (pp. 129-140). Leuven: Leuven University Press.

Thompson, B. (2004). Exploratory and Confirmatory Factor Analysis: Understanding Concepts and Applications. Washington, D.C.: American Psychological Association. 
Torres-Acosta, N. D., Rodríguez-Gómez, J. y Acosta-Vargas, M. (2013). Personalidad, aprendizaje y rendimiento académico en medicina. Investigación en Educación Médica, 2 (8), 193-201.

\section{Biografía de los autores}

Jordi Longás Mayayo es Profesor Asociado de la Facultad de Psicología, Ciencias de la Educación y del Deporte Blanquerna. Ha coordinado el I+D+i en torno al Éxito Educativo en Contextos de Vulnerabilidad y es director ejecutivo del Equipo de Dirección Científica del programa CaixaProinfancia de la Obra Social «la Caixa». Sus principales líneas de investigación son el éxito escolar, las redes socioeducativas, la gestión escolar y el bienestar docente.

Elena Carrillo Álvarez es profesora en los estudios de Grado de la Facultad de Ciencias de la Salud Blanquerna, Investigadora Principal del grupo de investigación Global Research on Wellbeing (GRoW) y miembro de la Academia Española de Nutrición y Dietética. Sus principales líneas de investigación son: la promoción de una alimentación saludable en diferentes grupos socioeconómicos y el desarrollo de redes de gobernanza socioe- ducativa que conectan agentes sociales, educativos y de salud, desde una perspectiva de determinantes de la salud.

Albert Fornieles Deu es Doctor en Psicología por la Universidad de Barcelona (1995) con licenciaturas en Psicología (UB 1989) y Antropología Social y Cultural (UNED 2006). Actualmente colabora con la Fundación Blanquerna en el proyecto "Investigación sobre éxito escolar en contextos de pobreza y vulnerabilidad social a nivel del Estado Español». También participa en el proyecto «Inseguridad alimentaria, discriminación por el peso, alteraciones alimentarias y bienestar psicológico en adolescentes españoles».

Jordi Riera i Romaní es Catedrático de Educación de la Universidad Ramón Llull, en la Facultad de Psicología, Ciencias de la Educación y del Deporte Blanquerna. Ha sido jefe de departamento de educación y Decano de la misma Facultad (1996-2004) y actualmente es Vicerrector de política académica y adjunto al Rector de la Universidad Ramón Llull, e Investigador Principal del Grupo de Investigación Consolidado de «Pedagogía, Sociedad e Innovación con el apoyo de las TIC (PSITIC)». 


\section{revista española de pedagogía año LXXVI, nº 269, enero-abril 2018 \\ Spanish Journal of Pedagogy \\ year LXXVI, n. 269, January-April 2018 \\ sumario *}

\section{table of contents **}

José Antonio lbáñez-Martín

75 años al servicio de la educación

75 years at the service of education

\section{Estudios}

\section{Studies}

David Menéndez Álvarez-Hevia

Aproximación crítica a la Inteligencia Emocional

como discurso dominante en el ámbito educativo

A critical approach to Emotional Intelligence

as a dominant discourse in the field of education

Javier Tourón, Deborah Martín,

Enrique Navarro Asencio, Silvia Pradas

y Victoria Î́nigo

Validación de constructo de un instrumento

para medir la competencia digital docente

de los profesores (CDD)

Construct validation of a questionnaire

to measure teachers' digital competence (TDC)
Jordi Longás Mayayo, Elena Carrillo Álvarez,

Albert Fornieles Deu y Jordi Riera i Romaní

3 Desarrollo y validación del cuestionario

sobre condicionantes de éxito escolar

en alumnos de secundaria

Development and validation of a questionnaire

about determinants of academic success

in secondary school students

7 Notas

Notes

Carmen Caro Samada, Josu Ahedo Ruiz

y Francisco Esteban Bara

La propuesta de educación moral

de Kohlberg y su legado en la universidad:

actualidad y prospectiva

Kohlberg's moral education proposal and

25 its legacy at university: present and future

\footnotetext{
* Todos los artículos están publicados en inglés en la página web de la revista: www.revistadepedagogia.org.

** All the articles are published in English on the web page of the journal: www.revistadepedagogia.org.
} 


\section{Jaume Camps Bansell y Elisabeth Vierheller}

Escuelas diferenciadas en España:

un análisis cualitativo de las razones

y percepciones de sus directivos

Single-sex schools in Spain: a qualitative analysis

of the reasoning and perceptions of their principals

\section{Pilar Martínez Clares y Cristina González Lorente}

Orientación, empleabilidad e inserción laboral

en la universidad a través de un

Modelo de Ecuaciones Estructurales

Career guidance, employability, and entering

the workforce at University through a Structural

Equation Model

\section{Olga Duarte Piña}

La Enseñanza de la Historia: innovación y continuidad desde Rafael Altamira

Teaching History: innovation and continuity

since Rafael Altamira

141

Josefina Sánchez Rodríguez,

Talia Cristina Morillo Lesme y

\section{Concepción Riera Quintana}

Evaluación de las necesidades afectivas en niñas y niños adoptados: manifestaciones en su expresividad psicomotriz

Evaluating the affective needs of adopted children: demonstrations of psychomotor expressiveness

101

\section{Reseñas bibliográficas}

Martínez, M., Esteban, F., Jover, G. y Payá, M.

La educación, en teoría (Juan Luis Fuentes).

Sarramona, J. Conservadores e izquierdistas frente a la educación (José Antonio Jordán).

Buxarrais, M. R. y Burget, M. (Coord.) Aprender

a ser. Por una pedagogía de la interioridad

(Gema Pilar Sáez Suanes). Musaio, M. Realizzo

me stesso. Educare i giovani alla ricerca delle possibilità (Carmen Urpí Guercia).

Pérez-Pérez, C. Educación en valores para la ciudadanía. Estrategias y técnicas de aprendizaje (José L. González-Geraldo).

\section{Informaciones}

Conferencia TEPE 2018 sobre «Reclutamiento y educación de los mejores docentes: política, profesionalismo y pedagogíay. I Congreso Internacional sobre «Educación del Carácter en Latinoamérica: Retos y Oportunidades». IX Congreso Internacional de Psicología y Educación (CIPE) sobre «Psicología, Educación y Neurociencias. Construyendo puentes para el desarrollo humano». V Congreso Internacional EDO (CIEDO) sobre «Liderazgo y gestión del talento en las organizaciones».

157 Una visita a la hemeroteca (Eva Ramírez Carpeño). Una visita a la red (David Reyero).

\section{Instrucciones para los autores}

Instructions for authors 201

ISSN 0034-9461 - Depósito legal: M. 6.020 - 1958 e-ISSN 2174-0909 Rev. esp. pedagog. (Internet) INDUSTRIA GRÁFICA ANZOS, S.L. Fuenlabrada - Madrid 